\title{
Abdominal imaging: Diagnosis
}

\section{S Beningfield, P Scholtz, S Misser}

Division of Radiology, Groote Schuur Hospital and University of Cape Town

S Beningfield, MB ChB, FFRad (D) SA

P Scholtz, MB ChB, FCRad (D) SA

Lake Smit and Partners, Durban

S Misser, MB ChB, FCRad (D) SA

Corresponding author: S Misser (misser@lakesmit.co.za)

We congratulate Dr Omar (details not to hand at time of going to press) for his close to spot-on diagnosis, for which he receives the award of R1 000 from the RSSA. Professor Beningfield et al. elaborate below on the condition and its imaging. Please refer to page 152 of the December 2012 issue of the SAJR (http://dx.doi.org/10.7196/SAJR.795) for the presentation details and unannotated images.

\section{Diagnosis}

The 40-year-old man underwent partial gastrectomy with Roux-en-Y gastrojejunostomy for gastro-oesophageal reflux unresponsive to fundoplication. Ten days after the operation, he began vomiting and also complained of epigastric pain. A CT scan (Figs 1, 2 and 3) was performed. The scout image (Fig. 1) demonstrated the presence of a post-operative drainage catheter (broad blue arrow), isolated distended loop of the small bowel, probably jejunum (thin blue arrow), as well as a fixed hiatus hernia in a gastric remnant (broad grey arrow). The final diagnosis was afferent loop obstruction in a patient following recent partial

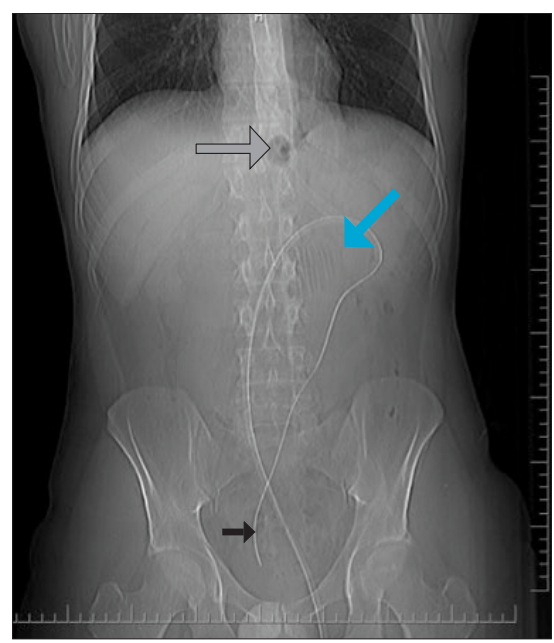

Fig. 1. Planning view showing surgical drain (thin arrow), isolated distended small bowel loop (broad blue arrow), and small hiatus hernia (broad grey arrow). gastrectomy and Roux-en-Y anastomosis, as well as a prior fundoplication. The Rouxen-Y anatomy was suggested by the lack of the afferent loop ascending to the stomach.

\section{Background}

Although first reported in 1881, afferent and efferent loop obstructions have been better understood since the $1950 \mathrm{~s}^{[1]}$ following the

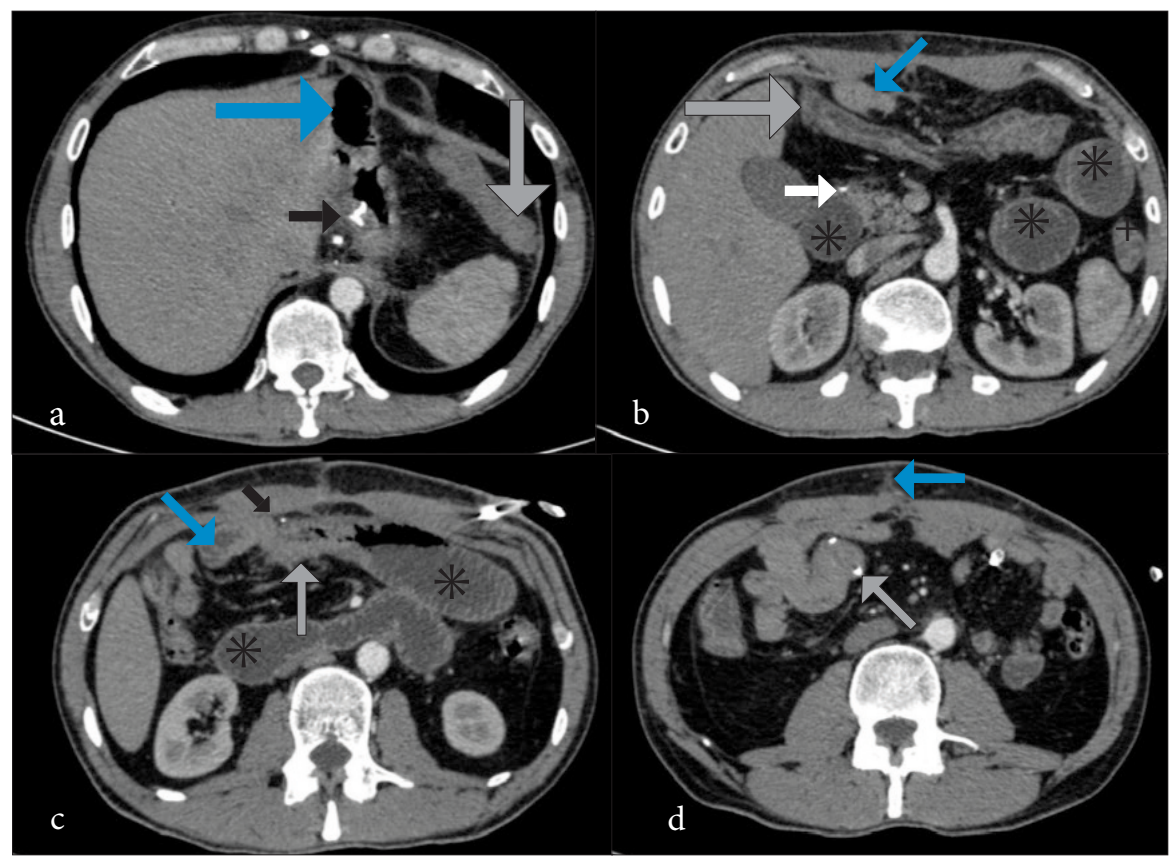

Fig. 2. Axial post-contrast CT images of the abdomen. (a) Fundoplication sutures (thin arrow), empty stomach (broad blue arrow) and collapsed splenic flexure of colon (broad grey arrow). (b) Suture in duodenal stump (thin blue arrow), distended duodeno-jejunal afferent limb $\left(^{*}\right)$, undistended pre-colic efferent limb from gastric remnant (broad blue arrow), collapsed transverse colon (broad grey arrow) and descending colon (+). (c) Distended duodeno-jejunal afferent limb $\left(^{*}\right)$ crossing between SMA and aorta and minimally distended pre-colic efferent limb (broad blue arrow) from gastric remnant, with transition point (broad grey arrow) in region of anastomotic staple (thin arrow). (d) Undistended gastrojejunostomy blind limb with sutures (broad grey arrow) and surgical scar (broad blue arrow), drain (thin arrow) and no other distended bowel. 


\section{QUIZ CASE}

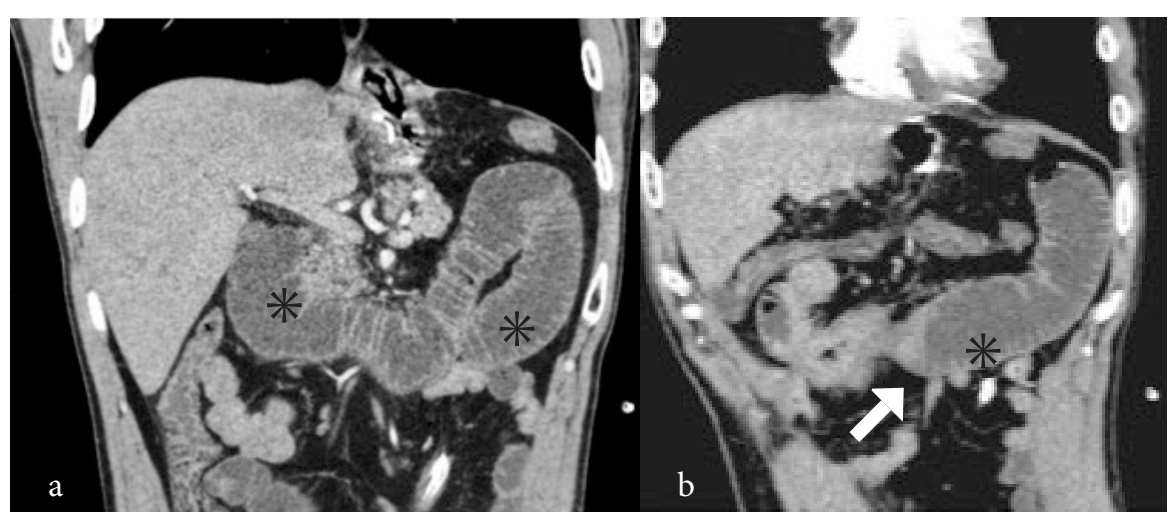

Fig. 3. $3 a$ and 3b: Coronal multiplanar reconstructions showing distended duodeno-jejunal afferent limb ${ }^{*}$ with transition point (arrow).

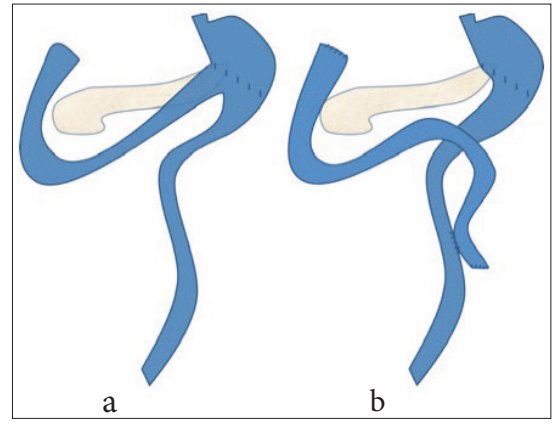

Fig. 4. (a) Bilroth II gastrectomy with gastrojejunostomy. (b) Partial gastrectomy with Rouxen-Y jejuno-jejunostomy anastomosis.

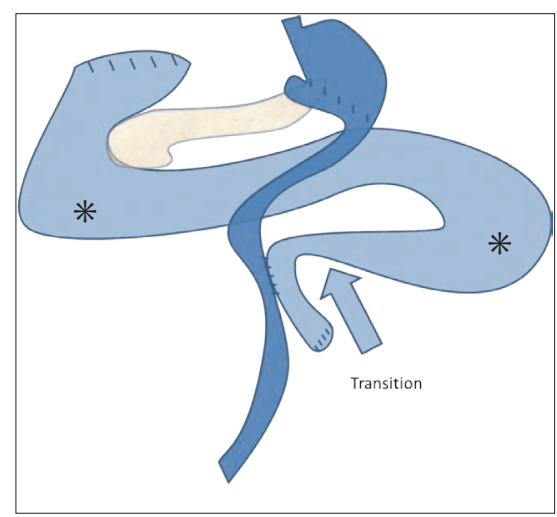

Fig. 5. Transition point (broad arrow) with distended afferent duodeno-jejunal $\operatorname{limb}\left({ }^{*}\right)$.

popularity of Billroth II gastrectomy with gastrojejunostomy (Fig. 4). While afferent means carrying inwards or toward a centre, efferent means carrying outward or away from a centre. The afferent loop syndrome is due to chronic partial obstruction causing distension of the proximal duodenum and jejunum, with discomfort and nausea. Afferent loop obstruction is a specific form of closed loop obstruction with the proximal component being the surgically closed-off duodenal stump (Figs 4 and 5), and the distal/downstream obstruction due to a variety of causes. Efferent loop obstruction is a form of high small bowel obstruction that may also divert gastric content into the afferent loop.

As Roux-en-Y anastomoses have become popular with partial gastrectomies and Whipple's procedures, they too are prone to both forms of obstruction around the jejuno-jejunal Roux-en-Y anastomosis (Figs $2 \mathrm{~b}$ and 5), possibly even more so. ${ }^{[2]}$ They also may develop a blind-pouch syndrome affecting the closed stump at the side-to-side anastomosis. ${ }^{[3]}$

\section{Frequency and causes}

The occurrence of afferent loop obstruction depends on length of follow-up, ranging between $0.2 \%$ and $20 \%{ }^{[4]}$ Where gastric carcinoma was the indication for the initial operation, ${ }^{[5]}$ tumour recurrence was noted to outweigh other usual causes such as adhesions, anastomotic ulcers, stenoses, internal hernias in surgically created foraminae (including in the mesentery), twisting foci, or intussusceptions. ${ }^{[2,6]}$

\section{Clinical presentation}

The non-specific symptoms classically occur either in the postoperative period or much later e.g. owing to tumour recurrence.

Epigastric pain owing to distension of the duodeno-jejunal loop dominates clinically. Vomiting, often without bile, is somewhat surprisingly found in $25 \%$ of cases and is thought to occur owing to sudden decompression of the afferent loop into the stomach; this is less plausible with the distance of the Roux-en-Y anastomosis from the stomach and may be related to nausea arising from loop distension. Biochemically, there may be slight elevations of amylase and obstructive liver enzymes.

\section{Diagnosis}

A clear understanding of the preceding surgical procedures and resultant anatomy facilitate interpretation of what may initially be confusing images. Plain films seldom provide helpful information as the loop is commonly fluid-filled. However, a small amount of gas in the loop, as in the case reported, can reveal distended valvulae conniventes. Oral contrast can occasionally reflux into the afferent loop.

Ultrasound has been used to detect the large fluid-filled C-loop between the SMA and aorta, with the presence of valvulae conniventes helpful in distinguishing these loops from acute pancreatic fluid collections, as pancreatitis often enters the differential diagnosis because of the pain and elevated amylase. $^{[4]}$ Biliary dilatation may also occasionally be noted.

CT in this condition was first reported in the $1980 \mathrm{~s},{ }^{[7]}$ and is now the preferred modality, particularly multislice CT. ${ }^{[2]}$ Oral contrast is helpful; IV contrast distinguishes vessel, nodes and tumour recurrence, as well as bowel viability. Coronal and sagittal views frequently clarify the anatomy (Fig. 3). The 'keyboard sign' produced by the valvulae conniventes is further supportive evidence. ${ }^{[5]}$ The presence of bowel ischaemia can be determined with arterial and venous phase $\mathrm{CT}$, and the detection the 'whirl sign' points to internal herniation. Seeking subtle features of adhesive obstruction such as anterior peritoneal thickening, small bowel loops closely applied to the anterior peritoneum, acute bowel loop kinks, fine mesenteric lines and bowel wall asymmetry may assist in complicated cases. ${ }^{[3]}$

MRI is also helpful where CT is not readily accessible, with MRCP an additional option. ${ }^{[6]}$

\section{Complications}

As a closed-loop obstruction, there is a risk of ischaemia and perforation owing to wall tension, with a mortality rate of up to $35 \%$ at 36 hours, so there is urgency in management. ${ }^{[3]}$

\section{Treatment}

The standard therapy is surgical decompression, often by means of enteroenterostomy, as was done in this case; this can also be achieved by laparoscopic or endoscopic intervention, and interventive radiology can offer temporary duodenojejunal decompression by means of internalexternal biliary drainage. 


\section{QUIZ CASE}

\section{Conclusion}

In the context of a patient with the appropriate surgical background (either given clinically or deduced by imaging evidence) presenting with epigastric discomfort, nausea or vomiting, the preferred imaging by multi-slice CT should allow accurate detection and rapid resolution of the afferent loop syndrome.

1. Warren RP. Acute obstruction of afferent or efferent loop, following antecolic partial gastrectomy, with report of three cases. Ann Surg 1954;139(2):202-205.

2. Juan Y-H, Yu C-Y, Hsu H-H, et al. Using multidetector-row CT for the diagnosis of afferent loop syndrome following gastroenterostomy reconstruction. Yonsei Med J 2011;52(4):574-580.
3. Sandrasegaran K, Maglinte DDT. Imaging of small bowel-related complications following major abdominal surgery. Eur J Radiol 2005;53(3):374-386.

4. Lee DH, Lim JH, Ko YT. Afferent loop syndrome: Sonographic findings in seven cases. Am J Roentgenol 1991;157(1):41-43.

5. Kim HC, Han JK, Kim KW, et al. Afferent loop obstruction after gastric cancer surgery: Helical CT findings. Abdom Imaging 2003;28(5):624-630.

6. Aoki M, Saka M, Morita S, Fukagawa T, Katai H. Afferent loop obstruction after distal gastrectomy with Roux-en-Y reconstruction. World J Surg 2010;34(10):2389-2392.

7. Gale ME, Gerzof SG, Kiser LC, et al. CT appearance of afferent loop obstruction. Am J Roentgenol 1982;138(6):1085-1088.

S Afr J Rad 2013;17(1):43-45. DOI:10.7196/SAJR.842 